\title{
Beyond control: applying ecological research to improve long-term outcomes of wilding conifer management
}

\author{
Ian A Dickie ${ }^{1}$, Joanna Green ${ }^{1}$, Duane A Peltzer ${ }^{2}$, Rowan Sprague ${ }^{3}$, Kate Orwin ${ }^{2}$, and \\ Sarah Sapsford ${ }^{1,4}$ \\ ${ }^{1}$ BioProtection Research Centre, School of Biological 8 Sciences, Te Kura Pūtaiao Koiora, \\ Te Whare Wānanga o Waitaha| University of Canterbury \\ ${ }^{2}$ Manaaki Whenua Landcare Research, Lincoln \\ ${ }^{3}$ New Zealand Wilding Conifer Group \\ ${ }^{4}$ Biosecurity New Zealand, Ministry for Primary Industries
}

June 16, 2021

\section{Hosted file}

Beyond control_2.pdf available at https://authorea.com/users/368780/articles/526372-beyondcontrol-applying-ecological-research-to-improve-long-term-outcomes-of-wilding-conifermanagement 